\title{
EfECTOS DE LA EXTRACCIÓN NO CONTROLADA DE MADERA SOBRE LA COMUNIDAD Y ESTRUCTURA DE TAMAÑOS de los manglares de Alvarado, Veracruz, México
}

\author{
María Teresa Rodríguez-Zúñiga', Pedro Ramírez-García ${ }^{2}$ y Gabriel Gutiérrez-Granados $3{ }^{3,4}$ \\ 'Subdirección de Percepción Remota, Comisión Nacional para el Conocimiento y Uso de la Biodiversidad \\ ${ }^{2}$ Laboratorio de Vegetación Acuática, Instituto de Biología, Universidad Nacional Autónoma de México \\ ${ }^{3}$ Laboratorio de Interacción Planta-Animal, Instituto de Ecología, Universidad Nacional Autónoma de México \\ ${ }^{4}$ Autor para la correspondencia: ggranados@ecologia.unam.mx
}

\begin{abstract}
Resumen: En este trabajo se analizó el efecto de la extracción no controlada de madera sobre la comunidad y estructura de tamaños de los manglares de Alvarado. Para realizar ésto se muestrearon 15 sitios de los cuales se obtuvo la composición florística, altura y área basal. Los efectos sobre la estructura de tamaños se evaluaron con un análisis de la distribución de individuos por categoría de tallas. Se documentó la presencia de Avicennia germinans, Laguncularia racemosa y Rhizophora mangle. Los parámetros estructurales fueron similares a los registrados en otros manglares de la zona tropical de México; sin embargo, los sitios con mayor influencia humana presentan cambios en la dominancia de especies. El análisis por categoría de tamaños indica que $L$. racemosa tiene problemas de regeneración debido principalmente a la extracción de individuos $<10 \mathrm{~cm}$ de diámetro de tallo. Los datos sugieren que de continuar con la extracción actual de tallos de L. racemosa, la situación local de la especie puede estar en riesgo.

Palabras clave: Avicennia germinans, categoría de tamaños diamétricos, extracción de madera, Laguncularia racemosa, Rhizophora mangle.

Abstract: We analyzed the effects of uncontrolled timber extraction on community and diameter-size of mangroves in Alvarado. We sampled 15 sites, gathering data on floristic composition, height, and basal area. Changes in diameter-size were evaluated through the size-distribution analysis. Avicennia germinans, Laguncularia racemosa, and Rhizophora mangle were present in the area. Structure parameters were similar to others documented in similar latitudes in Mexico, but the sites under human influence had shifts in species dominance. The analysis of diameter-size distributions indicated that $L$. racemosa was underrepresented due to logging, mainly of individuals $<10 \mathrm{~cm}$ in diameter. We suggest that current extraction trends threaten the distribution of $L$. racemosa.
\end{abstract}

Key words: Avicennia germinans, diameter-size categories, Laguncularia racemosa, Rhizophora mangle, timber extraction.

L os manglares ocupan al menos el $75 \%$ de la línea costera intertropical (Day et al., 1987; Osborne, 2000). Sin embargo, representan sólo el 1\% del área total de los bosques tropicales (Ellison et al., 1999) y están entre los ecosistemas más amenazados de los trópicos (Valiela et al., 2001; Duke et al., 2007). Esto es porque las presiones antropogénicas están reduciendo de manera alarmante la cobertura global de los manglares, ya sea directa o indirectamente como resultado de actividades de desarrollo piscícola, urbano, turístico, industrial o portuario (Farnsworth y Ellison, 1997; Barbier y Strand, 1998; Elster, 2000; Valiela et al., 2001).

Por otro lado, la extracción de árboles para leña, tapos, construcción de casas y manufactura de herramientas tam- bién representa un impacto para los manglares (ValdezHernández, 2002; Hernández-Cornejo et al., 2005; Walters, 2005a; Alongi y de Carvalho, 2008). La presión producida por el aprovechamiento del manglar puede conducir a cambios en la dinámica de la regeneración y estructura de un bosque de manglar (Benfield et al., 2005; Walters, 2005a). Además, cuando la extracción a pequeña escala es dirigida hacia las tallas pequeñas (diámetro $<10 \mathrm{~cm}$ ) de una especie o un grupo de especies se corre el riesgo de provocar una extinción local de las especies aprovechadas, ya que se limita su regeneración natural (Obiri et al., 2002; Lawes et al., 2007).

Los manglares tienen una historia de extracción generali- 
zada en todo el mundo (Alongi, 2002; Walters, 2005a; Duke et al., 2007). Sin embargo, los efectos a largo plazo de la remoción de individuos de diferentes tallas sobre la composición de la comunidad y la estructura poblacional apenas ha comenzado a ser analizada (Alongi y Carvalho, 2008; Feka et al., 2009). Se ha sugerido que puede utilizarse la distribución de tallas como indicador de la estabilidad poblacional de una especie (Condit et al., 1998), ya que teóricamente, las poblaciones con más juveniles con respecto los adultos son consideradas estables (una distribución log-normal), pero las que cuentan con pocos juveniles pueden enfrentar problemas de regeneración y su población está en declive poblacional (Hubbell y Foster, 1983; Lewis et al., 2009). Esta técnica ha servido para analizar las tendencias poblacionales de diferentes especies de árboles tropicales y de manera general representa la historia de la regeneración de ésta (Condit et al., 1998; Lawes et al., 2007). Así, un primer paso para entender la dinámica de una comunidad de manglar bajo un esquema de extracción no controlado es una caracterización de la estructura de tamaños de la comunidad al mismo tiempo que se analizan las características de la población como son la regeneración natural y aumento en talla de los individuos analizados (Boudreau et al., 2005). Nuestra hipótesis es que las comunidades de manglar sujetas a extracción de madera presentarán cambios en su estructura de tamaños y reclutamiento. Para tal fin, los objetivos de este trabajo fueron: (a) caracterizar la estructura (área basal, frecuencia y dominancia) y composición de los manglares de Alvarado, Veracruz, México y (b) determinar el impacto de la corta no controlada a pequeña escala sobre la estructura de tamaños del manglar.

\section{Métodos}

Zona de estudio. El Sistema Lagunar de Alvarado (SLA), se localiza en el centro del estado de Veracruz, en el Golfo de México (18 $\left.43^{\prime}-18^{\circ} 52^{\prime} \mathrm{N}, 95^{\circ} 45^{\prime}-95^{\circ} 57^{\prime} \mathrm{O}\right)$. El clima es cálido subhúmedo con lluvias abundantes en verano, con una temperatura media anual de $26.1^{\circ} \mathrm{C}$ y precipitación anual de $1,828 \mathrm{~mm}$ (CONAGUA, 2008). El sistema lagunar es alimentado fundamentalmente por el río Papaloapan, uno de los más caudalosos del país. El SLA es un humedal prioritario dentro de las Áreas de Importancia para Conservación de las Aves en México (AICA) y una región prioritaria marina de conservación nacional (Arriaga-Cabrera et al., 1998).

Estructura del bosque. La vegetación fue muestreada a través de 15 transectos dentro del SLA. La selección de los sitios se llevó a cabo con la ayuda de imágenes de satélite previamente analizadas en las que se clasificó el cambio de uso de suelo de la vegetación de la zona (Rodríguez-Zúñiga, 2002). Además se realizaron recorridos de campo en los que se registraron una serie de variables cualitativas que permitieron confirmar lo determinado por las imágenes de satélite

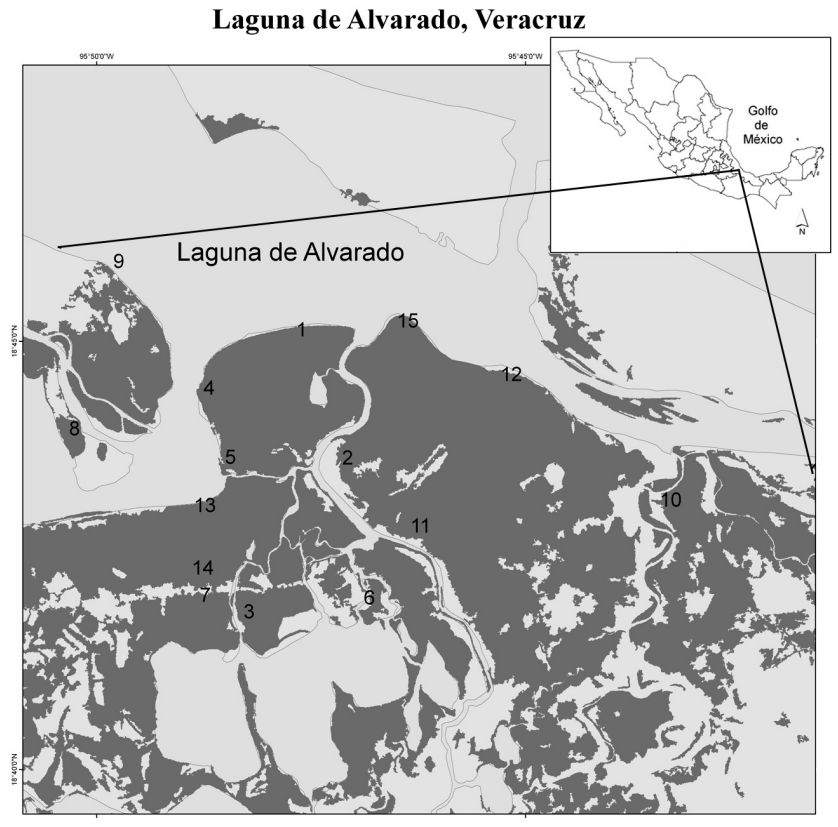

Figura 1. Localización de los sitios conservados $(4,5,7,8,11$, $12,13$ y 14$)$ y no conservados $(1,2,3,6,9,10$ y 15$)$, muestreados dentro del Sistema Lagunar de Alvarado, Veracruz.

(Cuadro 1). Así, se seleccionaron ocho sitios conservados $(4,5,7,8,11,12,13$ y 14$)$ y siete sitios no conservados (1, 2, 3, 6, 9, 10 y 15; Figura 1). La estructura del manglar fue determinada usando el método de Gentry (1982) con una modificación en la longitud de los transectos; $500 \mathrm{~m}$ de largo por $2 \mathrm{~m}$ de ancho $\left(1,000 \mathrm{~m}^{2}\right)$. En cada transecto se midieron a todos los individuos con un diámetro a la altura del pecho (DAP) $\geq 2.5 \mathrm{~cm}$, con el fin de incorporar a los individuos ya establecidos del contingente de la regeneración (Sherman et $a l ., 2000)$. De cada individuo fue medido el DAP y la altura con un flexometro $( \pm 1 \mathrm{~cm})$ para los individuos menores de $2 \mathrm{~m}$ y con un clisímetro $\left(1^{\circ} \pm 0.1^{\circ}\right.$, Rossbach) para los mayores a esta altura. La identidad específica fue asignada

Cuadro 1. Características cualitativas consideradas para la discriminación entre sitios conservados y no conservados en el Sistema Lagunar de Alvarado. SE, sin evidencia; $X$, evidencia minima; XX, evidencia media; XXX, evidencia alta.

\begin{tabular}{lcc}
\hline Característica & Conservado & $\begin{array}{c}\text { No } \\
\text { conservado }\end{array}$ \\
\hline Presencia de ganado & $\mathrm{SE}$ & $\mathrm{XXX}$ \\
Cercanía a vías fluviales principales & $\mathrm{X}$ & $\mathrm{XXX}$ \\
Presencia de casas a menos de 10 km & $\mathrm{SE}$ & $\mathrm{XXX}$ \\
Evidencia de corte de árboles & $\mathrm{X}$ & $\mathrm{XXX}$ \\
Presencia de desmontes & $\mathrm{SE}$ & $\mathrm{XXX}$ \\
Presencia de pastizal inducido & $\mathrm{X}$ & $\mathrm{XXX}$ \\
\hline
\end{tabular}


a todos los individuos. Con estos datos se calculó el área basal y los valores relativos de dominancia (DoR), frecuencia (FR) y densidad (DR) de cada especie (Cintrón-Molero y Schaeffer-Novelli, 1984). El valor de importancia (IV = $\mathrm{RDo}+\mathrm{RF}+\mathrm{RD}$ ) fue utilizado para evaluar la contribución relativa de cada especie dentro de la comunidad (Curtis y McIntosh, 1951). Dentro de los mismos transectos se midieron e identificaron todos los tocones con evidencia de corte.

Para analizar el efecto del corte de árboles sobre la estructura de tamaños se usó un análisis de la distribución de frecuencias del diámetro a la altura del pecho (DAP). Estos datos se compararon entre transectos con y sin evidencia de perturbación humana. A pesar de que este análisis es considerado estático y a corto plazo (Condit et al., 1998), provee información sobre la estabilidad de la población y el potencial de regeneración (Boudreau et al., 2005, Lawes et al., 2007).

Todos los individuos vivos y los tocones de cada una de las especies de manglar fueron asignados a las siguientes clases diamétricas: $2.5-5,5.1-10,10.1-15,15.1-20,20.1-25$, $25.1-30,30.1-40,40.1-50$ y $>50 \mathrm{~cm}$. Se usaron clases que aumentan progresivamente debido a que la densidad de tallos decrece conforme aumenta la talla de los individuos y esto balancea el número de integrantes entre clases diamétricas (Condit et al., 1998). La interpretación de la distribución de las clases diamétricas fue basada en la pendiente obtenida en la regresión para cada especie (Lawes et al., 2007). La distribución tipo I tiene una pendiente negativa y representa un mayor número de individuos en las clases de tamaño pequeño en relación a los de tamaño grande. En la distribución tipo II, la pendiente no es significativamente diferente de cero e indica una proporción similar de árboles pequeños y grandes. Las distribuciones tipo III y IV, con pendiente positiva, resultan de una mínima o nula presencia de individuos en la clase diamétrica de $2.5-5 \mathrm{~cm}$.

Análisis de datos. Todos los datos tuvieron una distribución normal (prueba de Shapiro-Wilks, $P<0.05$ ). En consecuencia se usó un análisis de varianza de un factor (ANOVA) para comparar el DAP, el área basal y la altura entre sitios y especies de manglar. Cada variable independiente fue analizada por separado. Adicionalmente se realizó un análisis de componentes principales (ACP) utilizando las variables estructurales obtenidas en cada uno de los sitios con el fin de conocer la contribución relativa de cada una de las especies de manglar por estado de conservación.

Para analizar la influencia de las actividades humanas sobre la estructura poblacional del manglar se empleó una serie de regresiones lineales. Se usó el límite superior de la categoría diamétrica como variable independiente y el número de individuos dentro de cada categoría como variable dependiente. Para realizar el análisis de regresión, los valores de frecuencia de las clases diamétricas se sujetaron a una transformación logarítmica $\ln \left(\mathrm{N}_{\mathrm{i}}+1\right.$, tomando en cuenta que algunas clases tienen cero individuos; Lawes et al., 2007). Una vez estimadas, las pendientes por especie y condición de conservación se compararon entre sí a través de un análisis de covarianza (Sokal y Rohlf, 1995). Además, se realizó un análisis de covarianza usando como variable categórica la condición de los transectos (conservado, no conservado) y como variables continuas el límite superior de la categoría diamétrica y el número de individuos dentro de cada categoría. Los mismos análisis se realizaron con los tocones. Todos los análisis fueron considerados estadísticamente significativos si $P<0.05$.

\section{Resultados}

Estructura y composición del manglar. En el Sistema Lagunar de Alvarado, Veracruz, se registraron siete especies arbóreas, tres de ellas (Avicennia germinans, Laguncularia racemosa y Rhizophora mangle) mangles verdaderos. Las otras cuatro especies están asociadas a zonas inundables: Guatteria sp., Lycium carolinianum, Pachira aquatica y Randia aculeata (Cuadro 2).

Se registró un total de 1,339 árboles con DAP $>2.5 \mathrm{~cm}$, con una densidad promedio de $965 \pm 261$ árboles/ha. Existieron diferencias significativas entre sitios con y sin evidencias de corte para el DAP $\left(F_{1,14}=4.67 ; P<0.04\right)$, el área ba$\operatorname{sal}\left(F_{1,14}=5.3 ; P<0.003\right)$ y la altura $\left(F_{1,14}=9.27 ; P<0.01\right)$.

Cuadro 2. Datos estructurales generales (frecuencia relativa, área basal, densidad y dominancia) de las especies arbóreas presentes en las comunidades de manglar y asociadas en el Sistema Lagunar de Alvarado, Veracruz.

\begin{tabular}{|c|c|c|c|c|}
\hline Especies & Frecuencia relativa & Área basal $\left(\mathbf{m}^{2}\right)$ & $\begin{array}{c}\text { Densidad } \\
\text { (individual/ha) }\end{array}$ & Dominancia \\
\hline Avicennia germinans (L.) Stearn & 28.8 & 26.6 & 696 & 62.5 \\
\hline Laguncularia racemosa C.F. Gaertn. & 28.8 & 10.4 & 671 & 24.4 \\
\hline Rhizophora mangle L. & 28.8 & 5.3 & 378 & 12.4 \\
\hline Pachira aquatica Aubl. & 5.8 & 0.3 & 17.2 & 0.7 \\
\hline Guatteria sp. & 3.9 & 0.004 & 5.3 & 0.01 \\
\hline Lycium carolinianum Walter & 1.9 & 0.02 & 2.7 & 0.05 \\
\hline Randia aculeata L. & 1.9 & 0.02 & 1.3 & 0.01 \\
\hline
\end{tabular}




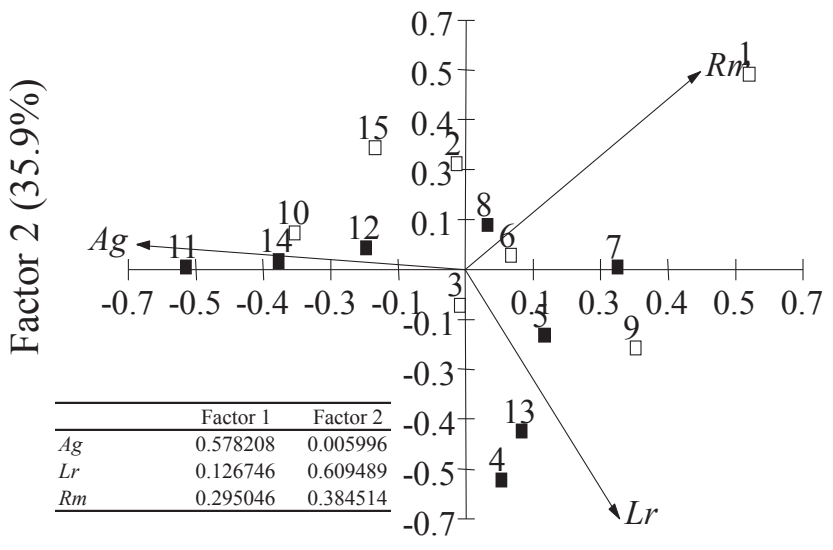

Factor $1(39.9 \%)$

Figura 2. Ordenación de los sitios en los dos primeros factores del análisis de componentes principales para los sitios conservados (cuadros negros) y no conservados (cuadros blancos). El cuadro en la figura muestra la contribución por especie a cada factor. Ag = Avicennia germinans $; \mathrm{Lr}=$ Laguncularia racemosa $; \mathrm{Rm}=$ Rhizophora mangle.
En cuanto a las especies de manglar Avicennia germinans fue la especie con la mayor área basal $\left(26.6 \mathrm{~m}^{2} / \mathrm{ha} ; F_{1,14}\right.$ $=23.3 ; P<0.001)$ y contribuyó con un $43 \%$ del valor de importancia de los sitios muestreados. El ACP indicó que la frecuencia de A. germinans y de Laguncularia racemosa fue mayor en los sitios conservados. Rhizophora mangle fue la especie más abundante en el sitio 1, pero estuvo presente en otros sitios sin importar su estado de conservación (Figura 2).

Influencia humana en la estructura de tamaños. En total se registraron 151 tocones de árboles extraídos por corte, lo que genera un resultado de 108 individuos extraídos por hectárea, la mayoría de ellos con un DAP $>5 \mathrm{~cm}$. Un mayor número de árboles de manglar (84\%) fueron extraídos en las zonas no conservadas $\left(F_{14,149}=23.3 ; P<0.001\right)$. Durante el periodo de estudio la especie que se extrajo con mayor frecuencia fue Laguncularia racemosa (Figura 3).

La figura 3 muestra los patrones en la distribución por

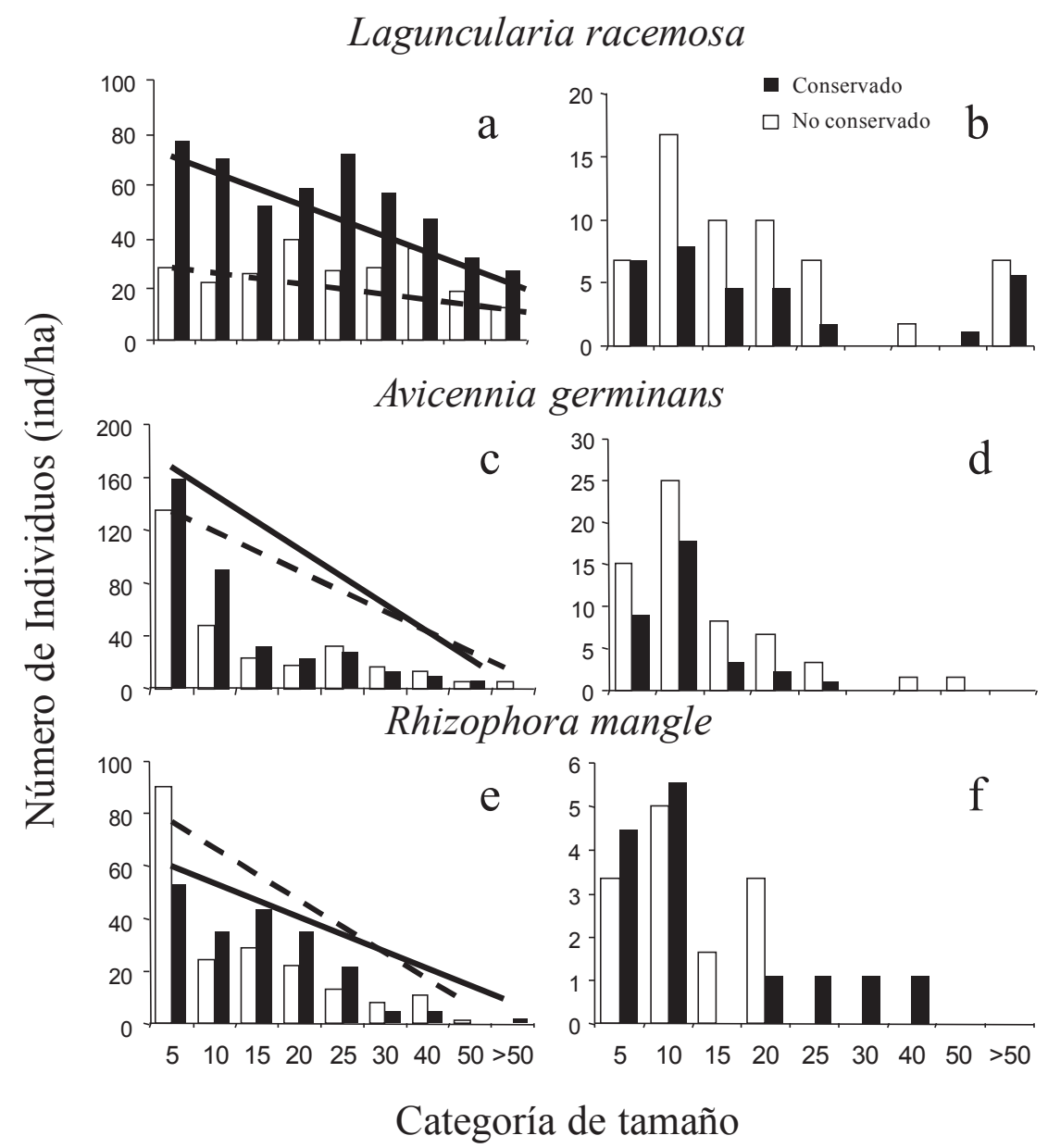

Figura 3. Clases diamétricas de los árboles vivos (a, c y e) y de los tocones (b, d, f) en sitios conservados (barras negras) y no conservados (barras blancas) de las tres especies de mangle registradas en el Sistema Lagunar de Alvarado. Las líneas punteadas muestran el ajuste de la regresión lineal para los sitios no conservados y las líneas continuas, el ajuste para las áreas conservadas. 
categoría diamétrica de los individuos de las tres especies de mangle analizados de acuerdo con el estado de conservación. En los sitios conservados la distribución de tamaños de Laguncularia racemosa mostró la forma de una $\mathrm{J}$ invertida (Tipo I), mientras que en áreas perturbadas, esta misma especie presentó una distribución de tamaños sin pendiente (Figura 3a; Tipo II). Además, existió una relación lineal negativa entre el número de individuos y la clase de DAP en los sitios no perturbados $\left(r^{2}=-0.81 ; F=35.3, P<\right.$ 0.01 ), mientras que en los sitios con perturbación no se presentó ninguna relación $\left(r^{2}=-0.31 ; F=3.2, P=0.11\right)$. Las pendientes entre ambas condiciones presentaron diferencias significativas $\left(F_{1,14}=19.3 ; P<0.01\right)$. El análisis de regresión indicó que esta variación en la distribución de tamaños fue explicada en un $78 \%$ por la perturbación humana. La distribución de los tocones en las diferentes categorías de tamaños en $L$. racemosa sugiere que los individuos en la categoría de $10 \mathrm{~cm}$ son los más extraídos. Sin embargo, la extracción en general está sesgada hacia los DAP $<25 \mathrm{~cm}$ $\left(F_{1,15}=4.9 ; P<0.04\right)$, tales diferencias se relacionan con las condiciones de conservación de los sitios (Figura $3 \mathrm{~b} ; \mathrm{F}_{1,15}=$ $18.1 ; P<0.01)$.

La distribución de tamaños de los individuos de Avicennia germinans fue similar en sitios conservados y no conservados, ajustándose a una pendiente de Tipo I $\left(r^{2}=-0.79\right.$; $F=31.4, P<0.01 ; r^{2}=-0.73 ; F=37.2, P<0.01$, respectivamente), sin diferencias significativas entre las pendientes $\left(F_{1,14}=3.1 ; P=0.09\right)$. La condición de los sitios no está asociada a la distribución de los tamaños $\left(F_{1,14}=1.06 ; P=\right.$ $0.31)$. Aunque hubo diferencias entre el número de tocones en cada categoría de tamaño $\left(F_{1,14}=15.1 ; P=0.01\right)$, éstas no estuvieron asociadas con la condición de los sitios (Figura $3 \mathrm{c}, \mathrm{d} ; F_{1,14}=1.5 ; P=0.22$ ).

En Rhizophora mangle, la distribución de frecuencias de categorías diamétricas fue de Tipo I tanto en sitios conservada $\left(r^{2}=0.85 ; F=40.2, P<0.01\right)$, como perturbados $\left(r^{2}\right.$ $=0.90 ; F=76.2, P<0.01)$. En esta especie tampoco hubo diferencias entre las pendientes $\left(F_{1,14}=0.01 ; P=0.97\right)$, ni efectos por el estado de conservación del sitio $\left(F_{1,14}=2.8 ; P\right.$ $=0.89)$. Por último, hay diferencias en el número de tocones en cada clase de tamaño $\left(F_{1,14}=16.24 ; P<0.01\right)$ pero no estuvieron asociadas al estado de conservación del sitio (Figura 3e,f; $F_{1,14}=0.03 ; P=0.85$ ).

\section{Discusión}

Los manglares del SLA están bajo constante presión humana, por lo que no es extraño que, como en otros sitios del mundo, la estructura y composición florística esté influenciada por las actividades directas e indirectas que se realizan en ellos (Walters, 2005a; Berger et al., 2006). Si bien el esfuerzo de muestreo es limitado, los transectos realizados muestran, en general, las características del Sistema Lagunar de Alvarado en cuanto a las condiciones de conservación analizadas. La estructura de los manglares del SLA presentó características de densidad, altura y área basal similares a lo documentado para otros bosques de mangles en México y el mundo (Lot-Helgueras et al., 1975; Pool et al., 1977; Harun-or-Rashid et al., 2009; Rakotomavo y Fromard, 2010). Sin embargo, la dominancia de las especies dependió de las características del sitio, pero sobre todo de la presión de corte que se hace sobre los manglares, ya que en sitios con características ambientales similares la dominancia cambia como resultado de la extracción (Walters, 2005a). Los sitios no conservados se ubican cerca de asentamientos humanos o son contiguos a lugares sometidos a cambios de uso de suelo, principalmente por ganadería. En estos sitios el número de árboles extraídos superó los 100 individuos por hectárea. Si consideramos que existen en promedio 956 árboles superiores a $2.5 \mathrm{~cm}$ de DAP en una hectárea de manglar, esta extracción correspondería al 10.5\% de los árboles del bosque, aunque hubo dos sitios no conservados en los que esto se incrementó hasta el $24 \%$.

Los sitios dominados por Avicennia germinans fueron aquellos con la influencia de ríos, lo que está asociado a una mayor cantidad de nutrientes y menor salinidad como ocurre con otras especies de manglar en el mundo (Aziz y Khan, 2001; Murray et al., 2003; McGowan et al., 2010). Rhizophora mangle fue la especie más abundante en un sólo sitio, el cual presenta una inundación permanente lo que es común para esta especie (Tomlinson, 1995), en los demás sitios fue registrada formando una franja en la interface entre tierra y agua. Un caso particular es el de Laguncularia racemosa, considerada como demandante de luz, por lo que su presencia probablemente está condicionada a la apertura de claros (Murray et al., 2003). Además en las zonas cercanas a los asentamientos humanos, esta especie presenta densidades bajas debido a la extracción como lo sugiere las categorías de tamaños de entre 5 - $25 \mathrm{~cm}$ que son las extraídas principalmente, lo que ha llevado a los manglares a tener una estructura y composición diferente entre las dos condiciones analizadas (Dahdouh-Guebas et al., 2000; Walters, 2005b; Alongi y Carvalho, 2008).

El análisis por categoría de tallas muestra que los eventos acumulados de corte a pequeña escala que realizan los pobladores de la zona de Alvarado ha cambiado la estructura de tamaños de Laguncularia racemosa. La falta de una estructura de tamaños que disminuya en el sentido de las categorías mayores (una pendiente negativa) y la distribución de tamaños discontinua de los individuos puede resultar en una extracción no sustentable de la especie (Boudreau et al., 2005; Lopez-Hoffman et al., 2006; Lawes et al., 2007) y que la estructura general del manglar puede estar modificada (Walters, 2005b; Alongi y Carvalho, 2008). Así, la extracción selectiva por especie y talla de especies de manglar puede comprometer el manejo futuro de los manglares (Barbier y Strand, 1998; Dahdouh-Guebas et al., 2000; Hernández-Cornejo et al., 2005; Berger et al., 2006), en el caso 
de Alvarado, con una menor representatividad del mangle blanco, Laguncularia racemosa. En contraste, como prácticamente no son utilizados, las poblaciones de Avicennia germinans y Rhizophora mangle cuentan con más individuos de tallas menores que de adultos, lo que sugiere que no están sujetas a la misma amenaza de disminución local de sus poblaciones (Lawes et al., 2007). Sin embargo, podrán ser utilizadas como recursos alternativos si la ausencia de individuos "cortables" de L. racemosa disminuye más (Dahdouh-Guebas et al., 2000).

En todo el SLA se requiere una evaluación sistemática de las tasas de extracción de los árboles de manglar, a fin de determinar si la regeneración natural de las tres especies documentadas es lo suficientemente rápida como para soportar las tasas actuales de extracción (Sherman et al., 2000). Se ha sugerido (Condit et al., 1998) que el análisis por tallas no representa la estructura de tamaños de una población en el sentido de que algunas categorías de tamaño no representan la edad del individuo. Sin embargo, los patrones generales de diferencias entre juveniles y adultos se mantienen (Condit et al., 1998). Inicialmente este tipo de análisis se realizó para bosques muy diversos, en donde no hay mucha correspondencia entre las especies del dosel y las del sotobosque. En bosques neotropicales de manglar hay pocas especies, por lo que la relación entre la composición florística entre individuos del dosel y el sotobosque es más estrecha (McGowan et al., 2010). Así, el análisis por tallas de la población es una opción viable para evaluar los cambios en la estructura poblacional de los manglares, aunque debe considerarse con precaución y validarse a largo plazo con poblaciones marcadas para tal fin.

Los resultados obtenidos en este trabajo documentan las consecuencias del manejo inadecuado de los manglares que reduce la abundancia de Laguncularia racemosa, pero la prohibición de la extracción de las especies de manglar no es aconsejable dadas las necesidades económicas de la zona además de que el uso de las diferentes especies de mangle está arraigado a las costumbres y cultura de los habitantes de Alvarado. La prohibición total ha probado ser ineficaz en países donde se realiza extracción forestal (Glaser et al., 2003, Hussain y Badola, 2010). La alternativa sería el manejo de los recursos forestales basado en la administración comunitaria de los recursos, que ha tenido éxito en la conservación y la restauración de manglares (Valdez-Hernández, 2004; Zorini et al., 2004; Walters, 2005b; López-Hoffman et al., 2006). Esto requiere el establecimiento y seguimiento de programas de extracción de madera con base en el conocimiento de la demografía de las especies de manglar para mantener el uso y conservar la comunidad vegetal.

\section{Agradecimientos}

El presente trabajo fue financiado por el Instituto de Biología y el Instituto Nacional de la Pesca. Agradecemos a Che- co, Oscar y Juan su participación en el trabajo de campo. TRZ estuvo becada por el CONACYT durante la realización del mismo. Asimismo, deseamos agradecer las observaciones de dos revisores anónimos, que mejoraron substancialmente el escrito.

\section{Literatura citada}

Alongi D.M. 2002. Present state and future of the world's mangrove forests. Environmental Conservation 29:331-349.

Alongi D.M. y Carvalho N.A. 2008. The effect of small-scale logging on stand characteristics and soil biogeochemistry in mangrove forests of Timor Leste. Forest Ecology and Management 255:1359-1366.

Arriaga-Cabrera L., Vázquez-Domínguez E., González-Cano J., Jiménez-Rosenberg R., Muñoz-López E. y Aguilar-Sierra V. Coords. 1998. Regiones Marinas Prioritarias de México. Comisión Nacional para el Conocimiento y uso de la Biodiversidad, México, D.F.

Aziz I. y Khan M.A. 2001. Experimental assessment of salinity tolerance of Ceriops tagal seedlings and saplings from the Indus delta, Pakistan. Aquatic Botany 70:259-268.

Barbier E.B. y Strand I. 1998. Valuing mangrove-fishery linkages A case study of Campeche, Mexico. Environmental \& Resource Economics 12:151-166.

Benfield S.L., Guzman H.M. y Mair J.M. 2005. Temporal mangrove dynamics in relation to coastal development in Pacific Panama. Journal of Environmental Management 76:263-276.

Berger U., Adams M., Grimm V. y Hildenbrandt H. 2006. Modelling secondary succession of Neotropical mangroves: causes and consequences of growth reduction in pioneer species. Perspectives in Plant Ecology, Evolution and Systematics 7:243-252.

Boudreau S., Lawes M.J., Piper S.E. y Phadima L.J. 2005. Subsistence harvesting of pole-size understorey species from Ongoye Forest Reserve, South Africa: species preference, harvest intensity, and social correlates. Forest Ecology and Management 216:149-165.

CONAGUA [Comisión Nacional de Agua] 2008. Estaciones Metereológicas Automáticas. Servicio Metereológico Nacional <smn.cna.gob.mx/emas/> (consultado diciembre 2010)

Cintrón-Molero G. y Schaeffer-Novelli Y. 1984. Methods for studying mangrove structure. En: Snedaker S.C. y Snedaker J.G. Eds. The Mangrove Ecosystem: Research Methods, pp. 91-131, UNESCO, Paris.

Condit R., Sukumar R., Hubbell S.P. y Foster R.B. 1998. Predicting population trends from size distributions: a direct test in a tropical tree community. The American Naturalist 152:495-509.

Curtis J.T. y McIntosh R.P. 1951. An upland forest continuum in the prairie-forest border region of Wisconsin. Ecology 32:476-496.

Dahdouh-Guebas F., Mathenge C., Kairo J.G. y Koedam N. 2000. Utilization of mangrove wood products around Mida Creek (Kenya) amongst subsistence and commercial users. Economic Botany 54:513-527.

Day J.W., Conner W.H., Ley-Lou F., Day R.H. y Machado-Navarro A. 1987. The productivity and composition of mangrove forests, Laguna de Terminos, Mexico. Aquatic Botany 27:267-284.

Duke N.C., Meynecke J.-O., Dittmann S., Ellison A.M., Anger K., Berger U., Cannicci S., Diele K., Ewel K.C., Field C.D., Koedam N., Lee S.Y., Marchand C., Nordhaus I. y Dahdouh-Guebas F. 
2007. A world without mangroves?. Science 317:41-42.

Ellison A.M., Farnsworth E.J. y Merkt R.E. 1999. Origins of mangrove ecosystems and the mangrove biodiversity anomaly. Global Ecology and Biogeography 8:95-115.

Elster C. 2000. Reasons for reforestation success and failure with three mangrove species in Colombia. Forest Ecology and Management 131:201-214.

Farnsworth E.J. y Ellison A.M. 1997. The global conservation status of mangroves. Ambio 26:328-334.

Feka N.Z., Chuyong G.B. y Ajonina G.N. 2009. Sustainable utilization of mangroves using improved fish-smoking systems: a management perspective from the Douala-Edea wildlife reserve, Cameroon. Tropical Conservation Science 2:450-468.

Gentry A.H. 1982. Patterns of Neotropical plant species diversity. Evolutionary Biology 15:1-84.

Glaser M., Berger U. y Macedo R. 2003. Local vulnerability as an advantage: mangrove forest management in Pará state, north Brazil, under conditions of illegality. Regional Environmental Change 3:162-172.

Harun-or-Rashid S., Biswas S.R., Böcker R. y Kruse M. 2009. Mangrove community recovery potential after catastrophic disturbances in Bangladesh. Forest Ecology and Management 257:923-930.

Hernández-Cornejo R., Koedam N., Ruiz-Luna A., Troell M. y Dahdouh-Guebas F. 2005. Remote sensing and ethnobotanical assessment of the mangrove forest changes in the NavachisteSan Ignacio-Macapule lagoon complex, Sinaloa, Mexico. Ecology and Society 10:16. <www.ecologyandsociety.org/vol10/ iss $1 / \operatorname{art} 16>$

Hubbell S.P. y Foster R.B. 1983. Diversity of canopy trees in a neotropical forest and implications for conservation. En: Sutton S.L., Whitmore T.C. y Chadwick A.C. Eds. Tropical Rain Forest: Ecology and Management, pp. 25-41. Blackwell, Oxford.

Hussain S.A. y Badola, R. 2010 Valuing mangrove benefits: contribution of mangrove forests to local livelihoods in Bhitarkanika Conservation Area, east coast of India. Wetlands Ecology and Management 18:321-331.

Lawes M.J., Griffiths M.E. y Boudreau S. 2007. Colonial logging and recent subsistence harvesting affect the composition and physiognomy of a podocarp dominated Afrotemperate forest. Forest Ecology and Management 247:48-60.

Lewis S.L., Lloyd J., Sitch S., Mitchard E.T.A. y Laurance W.F. 2009. Changing ecology of tropical forests: evidence and drivers. Annual Review of Ecology, Evolution, and Systematics 40:529-549.

López-Hoffman L., Monroe I.E., Narváez E., Martínez-Ramos M. y Ackerly D.D. 2006. Sustainability of mangrove harvesting: how do harvesters' perceptions differ from ecological analysis? Ecology and Society 11:14. <www.ecologyandsociety.org/ vol11/iss2/art14/>

Lot-Helgueras A., Vázquez-Yánez C. y Menendez F.L. 1975. Physiognomic and floristic changes near the northern limit of mangroves in the Gulf Coast of Mexico. En: Walsh G.E., Snedaker S.C. y Teas H.T. Eds. Proceedings of the International
Symposium on Biology and Management of Mangroves, pp. 5261. Instittute of Food and agricultural Sciences, University of Florida, Gainesville.

McGowan T., Cunningham S.L., Guzmán H.M., Mair J.M., Guevara J.M. y Betts T. 2010. Mangrove forest composition and structure in Las Perlas Archipelago, Pacific Panama. Revista de Biología Tropical 58:857-869.

Murray M.R., Zisman S.A., Furley P.A., Munro D.M., Gibson J., Ratter J., Bridgewater S., Minty C.D. y Place C.J. 2003. The mangroves of Belize: Part 1. distribution, composition and classification. Forest Ecology and Management 174:265-279.

Obiri J., Lawes M. y Mukolwe M. 2002. The dynamics and sustainable use of high-value tree species of the coastal Pondoland forests of the Eastern Cape Province, South Africa. Forest Ecology and Management 166:131-148.

Osborne P.L. 2000. Tropical Ecosystems and Ecological Concepts. Cambridge University Press, Cambridge.

Pool D.J., Snedaker S.C. y Lugo A.E. 1977. Structure of mangrove forests in Florida, Puerto Rico, Mexico, and Costa Rica. Biotropica 9:195-212.

Rakotomavo A. y Fromard F. 2010. Dynamics of mangrove forests in the Mangoky River delta, Madagascar, under the influence of natural and human factors. Forest Ecology and Management 259:1161-1169.

Rodríguez-Zúñiga M.T. 2002. Manglares del sistema lagunar de Alvarado, Ver. México: influencia de algunos procesos físicos, biológicos y antropogénicos en su distribución y estructura. Tesis de Maestría en Ciencias Biológica (Biología Ambiental) Facultad de Ciencias, Posgrado en Ciencias Biológicas, Universidad Nacional Autónoma de México, México, D.F. 104 pp.

Sherman R.E., Fahey T.J. y Battles J.J. 2000. Small-scale disturbance and regeneration dynamics in a neotropical mangrove forest. Journal of Ecology 88:165-178.

Sokal R.R. y Rohlf F.J. 1995. Biometry: The Principles and Practices of Statistics in Biological Research. Freeman, Nueva York.

Tomlinson P.B. 1995. The Botany of Mangroves. Cambridge University Press, Cambridge.

Valdez-Hernández J.I. 2002. Aprovechamiento forestal de manglares en el estado de Nayarit, costa Pacífica de México. Madera y Bosques 8:129-145.

Valdez-Hernández J.I. 2004. Manejo forestal de un manglar al sur de Marismas Nacionales, Nayarit. Madera y Bosques 10:93-104.

Valiela I., Bowen J.L. y York J.K. 2001. Mangrove forests: one of the world's threatened major tropical environments. Bioscience 51:807-815.

Walters B.B. 2005a. Ecological effects of small-scale cutting of Philippine mangrove forests. Forest Ecology and Management 206:331-348.

Walters B.B. 2005b. Patterns of local wood use and cutting of Philippine mangrove forests. Economic Botany 59:66-76.

Zorini L.O., Contini C., Jiddawi N., Ochiewo J., Shunula J. y Cannicci S. 2004. Participatory appraisal for potential communitybased mangrove management in East Africa. Wetlands Ecology and Management 12:87-102.

Recibido: 18 de diciembre de 2010

Aceptado : 18 de abril de 2011 